\title{
Correction to: Septal bowing and pulmonary artery diameter on computed tomography pulmonary angiography are associated with short-term outcomes in patients with acute pulmonary embolism
}

\author{
Mads Dam Lyhne ${ }^{1,2}$. Jacob Gammelgaard Schultz ${ }^{1,2}$ Peter J. MacMahon ${ }^{3}$. Faris Haddad ${ }^{4}$ Mannudeep Kalra ${ }^{5}$. \\ David Mai-King Tso ${ }^{5} \cdot$ Alona Muzikansky $^{6} \cdot$ Michael H. Lev $^{5} \cdot$ Christopher Kabrhel $^{1}$
}

Published online: 29 August 2019

(C) American Society of Emergency Radiology 2019

Correction to: Emergency Radiology (2019)

https://doi.org/10.1007/s10140-019-01709-9

The above article was published online with an error in an author's last name: It should be Muzikansky (and not Muzikanski). The correct name is presented here. The original article has been corrected.

Publisher's note Springer Nature remains neutral with regard to jurisdictional claims in published maps and institutional affiliations.

The online version of the original article can be found at https://doi.org/ 10.1007/s10140-019-01709-9

Christopher Kabrhel

ckabrhel@partners.org

1 Center for Vascular Emergencies, Department of Emergency Medicine, Massachusetts General Hospital, 55 Fruit Street,

Boston, MA 02114, USA

2 Department of Cardiology, Aarhus University Hospital, Palle Juul Jensens Boulevard 99, Aarhus N, 8200 Aarhus, Denmark

3 Department of Radiology, Mater Misericordiae University Hospital, Eccles Street, Dublin 7 Dublin, Ireland

4 Department of Radiology, Inova Fairfax Hospital, 3300 Gallows Road, Falls Church, VA 22042, USA

5 Department of Radiology, Massachusetts General Hospital, 55 Fruit Street, Boston, MA 02114, USA

6 Biostatistics Center, Massachusetts General Hospital, 50 Staniford Street, Boston, MA 02114, USA 